\title{
GENERALIZATIONS OF ALZER'S AND KUANG'S INEQUALITY
}

\author{
FENG QI
}

\begin{abstract}
Let $f$ be a strictly increasing convex (or concave) functions on $(0,1]$, then, for $k$ being a nonnegative integer and $n$ a natural number, the sequence $\frac{1}{n} \sum_{i=k+1}^{n+k} f\left(\frac{i}{n+k}\right)$ is decreasing in $n$ and $k$ and has a lower bound $\int_{0}^{1} f(t) d t$. Form this, some new inequalities involving $\sqrt[n]{(n+k) ! / k !}$ are deduced. By the Hermie-Hadamard inequality, several inequalities are obtained.
\end{abstract}

\section{Introduction}

In [1], H. Alzer, using the mathematical induction and other techniques, proved that for $r>0$ and $n \in \mathbb{N}$,

$$
\frac{n}{n+1} \leq\left(\frac{1}{n} \sum_{i=1}^{n} i^{r} / \frac{1}{n+1} \sum_{i=1}^{n+1} i^{r}\right)^{1 / r}<\frac{\sqrt[n]{n !}}{\sqrt[n+1]{(n+1) !}}
$$

By the Cauchy's mean-value theorem and the mathematical induction, the author in [7] presented that, if $n$ and $m$ are natural numbers, $k$ is a nonnegative integer, $r>0$, then

$$
\frac{n+k}{n+m+k}<\left(\frac{1}{n} \sum_{i=k+1}^{n+k} i^{r} / \frac{1}{n+m} \sum_{i=k+1}^{n+m+k} i^{r}\right)^{1 / r} .
$$

The lower bound is best possible.

From the Stirling's formula, for all nonnegative integers $k$ and natural numbers $n$ and $m$, the author in [8] obtained

$$
\left(\prod_{i=k+1}^{n+k} i\right)^{1 / n} /\left(\prod_{i=k+1}^{n+m+k}\right)^{1 /(n+m)} \leq \sqrt{\frac{n+k}{n+m+k}} .
$$

Received August 16, 1999.

2000 Mathematics Subject Classification. Primary 26D15.

Key words and phrases. Alzer's inequality, Kuang's inequality, convex function, HermiteHadamard inequality.

The author was supported in part by NSF of Henan Province (no. 004051800), SF for Pure Research of the Education Committee of Henan Province (no. 1999110004), and Doctor Fund of Jiaozuo Institute of Teachnology, The People's Republic of China. 
Let $f$ be a strictly increasing convex (or concave) function in $(0,1]$, J.-C. Kuang in [2] verified that

$$
\frac{1}{n} \sum_{k=1}^{n} f\left(\frac{k}{n}\right)>\frac{1}{n+1} \sum_{k=1}^{n+1} f\left(\frac{k}{n+1}\right)>\int_{0}^{1} f(x) d x .
$$

The study of Alzer's and Minc-Sathre's inequality has many literature, for examples, [1]-[9].

In this article, motivated by [2, 7], i.e. the inequalities in (2), (3) and (4), considering the convexity of a function, we get

Theorem 1. Let $f$ be strictly increasing convex (or concave) function in $(0,1]$, then the sequence $\frac{1}{n} \sum_{i=k+1}^{n+k} f\left(\frac{i}{n+k}\right)$ is decreasing in $n$ and $k$ and has a lower bound $\int_{0}^{1} f(t) d t$, that is,

$$
\frac{1}{n} \sum_{i=k+1}^{n+k} f\left(\frac{i}{n+k}\right)>\frac{1}{n+1} \sum_{i=k+1}^{n+k+1} f\left(\frac{i}{n+k+1}\right)>\int_{0}^{1} f(t) d t
$$

where $k$ is a nonnegative integer, $n$ a natural number.

If let $f(x)=x^{r}, r>0$, or let $k=0$ in (5), then the inequalities in (1), (2) and (4) could be deduced. Therefore, inequality (5) generalizes Alzer's and Kuang's inequality in $[1,2]$ and inequality (2) above.

Corollary 1. For a nonnegative integer $k$ and a natural number $n>1$, we have

$$
\begin{aligned}
& \frac{n+k}{n+k+1}<\left[\frac{(2 n+2 k) !}{(n+2 k) !}\right]^{1 / n} /\left[\frac{(2 n+2 k+2) !}{(n+2 k+1) !}\right]^{1 /(n+1)} \\
< & {\left[\frac{(n+k) !}{k !}\right]^{1 / n} /\left[\frac{(n+k+1) !}{k !}\right]^{1 /(n+1)}<\left[\frac{k !(k+2) !}{(k+3)^{2}}\right]^{1 / n(n+1)} . }
\end{aligned}
$$

For a larger $n$, the upper bound in the third inequality of (6) is not better than (3) for $m=1$. From the Hermite-Hadamard inequality in [3] and [4, pp. 10-12], we get the following

Theorem 2. Let $f$ be a nonlinear convex function in $(0,1]$, then

$$
\begin{aligned}
& \frac{1}{n+k} \sum_{i=k+1}^{n+k}\left[f\left(\frac{i}{n+k}\right)-f\left(\frac{2 i-1}{2(n+k)}\right)\right] \\
> & \frac{1}{n+k} \sum_{i=k+1}^{n+k} f\left(\frac{i}{n+k}\right)-\int_{k /(n+k)}^{1} f(t) d t \\
> & \frac{1}{2(n+k)}\left[f(1)-f\left(\frac{k}{n+k}\right)\right] .
\end{aligned}
$$

Further, if $f$ satisfies the Lipschitz condition

$$
|f(x)-f(y)| \leq M|x-y|^{\alpha}, \quad 0<\alpha \leq 1,
$$


then

$$
\frac{n}{n+k} \cdot \frac{M}{[2(n+k)]^{\alpha}}>\frac{1}{n+k} \sum_{i=k+1}^{n+k} f\left(\frac{i}{n+k}\right)-\int_{k /(n+k)}^{1} f(t) d t .
$$

If let $k=0$ in Theorem 2, the related result in [2] follows.

\section{Proof of Theorems}

Proof of Theorem 1. Let us first assume that $f$ be a strictly increasing convex function. Taking $x_{1}=\frac{i-1}{n+k}, x_{2}=\frac{i}{n+k}, \alpha=\frac{i-k-1}{n}$ and using the convexity and monotonicity of $f$ yields

$$
\begin{aligned}
& \frac{i-k-1}{n} f\left(\frac{i-1}{n+k}\right)+\left(1-\frac{i-k-1}{n}\right) f\left(\frac{i}{n+k}\right) \\
\geq & f\left(\frac{i-k-1}{n} \cdot \frac{i-1}{n+k}+\frac{n-i+k+1}{n} \cdot \frac{i}{n+k}\right) \\
= & f\left(\frac{n i-i+k+1}{n(n+k)}\right) \\
> & f\left(\frac{i}{n+k+1}\right)
\end{aligned}
$$

for $i=k+1, k+2, \ldots, n+k$. Summing up leads to

$$
\begin{aligned}
& \sum_{i=k+1}^{n+k}\left[\frac{i-k-1}{n} f\left(\frac{i-1}{n+k}\right)+\left(1-\frac{i-k-1}{n}\right) f\left(\frac{i}{n+k}\right)\right]>\sum_{i=k+1}^{n+k} f\left(\frac{i}{n+k+1}\right), \\
& \sum_{i=k+1}^{n+k}\left[(i-k-1) f\left(\frac{i-1}{n+k}\right)+(n+k-i+1) f\left(\frac{i}{n+k}\right)\right]>n \sum_{i=k+1}^{n+k} f\left(\frac{i}{n+k+1}\right), \\
& n \sum_{i=k+1}^{n+k} f\left(\frac{i}{n+k+1}\right)+n f(1)<(n+1) \sum_{i=k+1}^{n+k} f\left(\frac{i}{n+k}\right), \\
& n \sum_{i=k+1}^{n+k+1} f\left(\frac{i}{n+k+1}\right)<(n+1) \sum_{i=k+1}^{n+k} f\left(\frac{i}{n+k}\right) .
\end{aligned}
$$

The inequality (5) is proved.

By similar procedure, if $f$ is a strictly increasing concave function in $(0,1]$, then for $k<i \leq n+k$, we have

$$
\begin{aligned}
& \frac{i-k}{n+1} f\left(\frac{i+1}{n+k+1}\right)+\frac{n+k-i+1}{n+1} f\left(\frac{i}{n+k+1}\right) \\
\leq & f\left(\frac{i-k}{n+1} \cdot \frac{i+1}{n+k+1}+\frac{n+k-i+1}{n+1} \cdot \frac{i}{n+k+1}\right) \\
= & f\left(\frac{n i+2 i-k}{(n+1)(n+k+1)}\right)<f\left(\frac{i}{n+k}\right),
\end{aligned}
$$




$$
\begin{aligned}
& \sum_{i=k+1}^{n+k}\left[\frac{i-k}{n+1} f\left(\frac{i+1}{n+k+1}\right)+\frac{n+k-i+1}{n+1} f\left(\frac{i}{n+k+1}\right)\right] \\
= & \frac{n}{n+1} \sum_{i=k+1}^{n+k} f\left(\frac{i}{n+k+1}\right)+\frac{n}{n+1} f(1)<\sum_{i=k+1}^{n+k} f\left(\frac{i}{n+k}\right), \\
& n \sum_{i=k+1}^{n+k+1} f\left(\frac{i}{n+k+1}\right)<(n+1) \sum_{i=k+1}^{n+k} f\left(\frac{i}{n+k}\right) .
\end{aligned}
$$

The proof is complete.

Proof of Corollary 1. Substituting $f$ be $\ln (1+x)$ or by $\ln (x /(1+x))$ in (5) and simplifying yields the first or the second inequality in (6), respectively.

Since

$$
\begin{aligned}
\frac{[(n+k) ! / k !]^{n+1}}{[(n+k+1) ! / k !]^{n}} & =\sum_{j=3}^{n}\left\{\frac{[(j+k) ! / k !]^{j+1}}{[(j+k+1) ! / k !]^{j}}-\frac{[(j+k-1) ! / k !]^{j}}{[(j+k) ! / k !]^{j-1}}\right\}+\frac{[(k+2) ! / k !]^{3}}{[(k+3) ! / k !]^{2}} \\
& <\frac{k !(k+2) !}{(k+3)^{2}}
\end{aligned}
$$

the third inequality in (6) is obtained.

Proof of Theorem 2. Using the Hermite-Hadamard inequality in [3] and [4, pp. 10-12], we have

$$
\begin{aligned}
& \sum_{i=k+1}^{n+k} f\left(\frac{2 i-1}{2(n+k)}\right) \\
< & (n+k) \sum_{i=k+1}^{n+k} \int_{(i-1) /(n+k)}^{i /(n+k)} f(x) d x \\
< & \frac{1}{2} \sum_{i=k+1}^{n+k}\left[f\left(\frac{i}{n+k}\right)+f\left(\frac{i-1}{n+k}\right)\right] \\
= & \sum_{i=k+1}^{n+k} f\left(\frac{i}{n+k}\right)-\frac{1}{2}\left[f(1)-f\left(\frac{k}{n+k}\right)\right]
\end{aligned}
$$

that is

$$
\begin{aligned}
& \frac{1}{n+k} \sum_{i=k+1}^{n+k} f\left(\frac{2 i-1}{2(n+k)}\right)<\int_{k /(n+k)}^{1} f(x) d x \\
< & \frac{1}{n+k} \sum_{i=k+1}^{n+k} f\left(\frac{i}{n+k}\right)-\frac{1}{2(n+k)}\left[f(1)-f\left(\frac{k}{n+k}\right)\right] .
\end{aligned}
$$


The inequality (7) is proved. Combining (8) with (7) yields inequality (9). The proof of theorem 2 is complete.

\section{References}

[1] H. Alzer, On an inequality of H. Minc and L. Sathre, J. Math. Anal. Appl., 179(1993), 396-402.

[2] Ji-Chang Kuang, Some extensions and refinements of Minc-Sathre inequality, Math. Gaz., 83(1999), 123-127.

[3] Ji-Chang Kuang, Applied Inequalities, 2nd edition, Hunan Education Press, Changsha, China, 1993. (Chinese)

[4] D. S. Mitrinović, J. E. Pečarić and A. M. Fink, Classical and New Inequalities in Analysis, Kluwer Academic Publishers, Dordrecht/Boston/London, 1993.

[5] Feng Qi, An algebraic inequality, RGMIA Research Report Collection, 2(1999), 81-83. http://rgmia.vu.edu.au/v2n1.html

[6] Feng Qi and Lokenath Debnath, On a new generalization of Alzer's inequality, Intern. J. Math. \& Math. Sci., 25(2000), in the press.

[7] Feng Qi, Generalization of H. Alzer's inequality, Journal of Mathematical Analysis and Applications, 240(1999), 294-297.

[8] Feng Qi,Inequalities and monotonicity of sequences involving $\sqrt[n]{(n+k) ! / k !}$, RGMA Research Report College 2(1999). http://rgmia.vu.edu.au/v2n5.html

[9] Feng Qi and Qiu-Ming Luo, Generalization of H. Minc and J. Sathre's inequality, Tamkang Journal of Mathematics, 31(2000), 145-148.

Department of Mathematics, Jiaozuo Institute of Technology, \#142, Mid-Jiefang Road, Jiaozuo City, Henan 454000, The People's Republic of China.

E-mail: qifeng@jzit.edu.cn

URL: http://rgmia.vu.edu.au/qi.html 